\title{
Review
}

\section{Anti-tumor activities of active ingredients in Compound Kushen Injection}

\author{
Wei WANG ${ }^{1,2,3}$, Rong-li YOU ${ }^{1,4}$, Wen-jie QIN ${ }^{1,}{ }^{*}$, Li-na HAI ${ }^{1}$, Ming-jing FANG ${ }^{1}$, Guo-hua HUANG ${ }^{5}$, Rui-xia KANG ${ }^{4}$, Ming-hua $\mathrm{LI}^{2}$, \\ Yu-feng $\mathrm{QIAO}^{2}$, Jian-wei $\mathrm{LI}^{2,6}$, An-ping $\mathrm{LI}^{2, *}$ \\ ${ }^{1}$ Beijing Zhendong Guangming Pharmaceutical Research Institute Co Ltd, Beijing 100120, China; ${ }^{2}$ Shanxi Zhendong Pharmaceutical \\ Co Ltd, Changzhi 047100, China; ${ }^{3}$ Gastroenterology Department, the Second People's Hospital of Zhuhai, Zhuhai 519000, China; \\ ${ }^{4}$ Institute of Basic Research in Clinical Medicine, China Academy of Chinese Medical Sciences, Beijing 100700, China; ${ }^{5}$ Department \\ of Respiration, Nanfang Hospital of Southern Medical University, Guangzhou 510515, China; ${ }^{6}$ Department of Pharmacy, Chang Zhi \\ Medical College, Changzhi 046000, China
}

\begin{abstract}
Kushen (Radix Sophorae Flavescentis) has a long history of use for the treatment of tumors, inflammation and other diseases in traditional Chinese medicine. Compound Kushen Injection (CKI) is a mixture of natural compounds extracted from Kushen and Baituling (Rhizoma Smilacis Glabrae). The main principles of CKI are matrine (MT) and oxymatrine (OMT) that exhibit a variety of pharmacological activities, including anti-inflammatory, anti-allergic, anti-viral, anti-fibrotic and cardiovascular protective effects. Recent evidence shows that these compounds also produce anti-cancer actions, such as inhibiting cancer cell proliferation, inducing cell cycle arrest, accelerating apoptosis, restraining angiogenesis, inducing cell differentiation, inhibiting cancer metastasis and invasion, reversing multidrug resistance, and preventing or reducing chemotherapy-and/or radiotherapy-induced toxicity when combined with chemotherapeutic drugs. In this review, we summarize recent progress in studying the anti-cancer activities of MT, OMT and $\mathrm{CKI}$ and their potential molecular targets, which provide clues and references for further study.
\end{abstract}

Keywords: traditional Chinese medicine; Compound Kushen Injection; matrine; oxymatrine; anti-cancer drug

Acta Pharmacologica Sinica (2015) 36: 676-679; doi: 10.1038/aps.2015.24; published online 18 May 2015

\section{Introduction}

There is a long history of the use of traditional Chinese medicines (TCMs) to treat solid tumors in China ${ }^{[1]}$. In the past several decades, many TCMs have been approved by the Chinese State Food and Drug Administration and used in the clinical treatment of various types of solid tumors ${ }^{[2]}$. An increasing number of anti-cancer agents have been identified from the study of TCMs, although most of their molecular mechanisms are still waiting elucidation.

TCMs have been widely used in combination with chemotherapy or radiotherapy to improve the efficacy of cancer therapy and reduce side effects and complications ${ }^{[3]}$. Many years of clinical trials in China have shown that TCMs can modulate immune function and improve the quality of life of cancer patients, particularly for patients undergoing che-

\footnotetext{
* To whom correspondence should be addressed.

E-mail 229987727@qq.com (An-ping LI); 604928225@qq.com (Wen-jie QIN)

Received 2014-08-29 Accepted 2015-03-10
}

motherapy ${ }^{[4]}$. Most TCM formulas are mixtures of different natural compounds, which might function in different ways and target different molecular pathways ${ }^{[5]}$. An emerging approach in biomedical research, systems biology, is a systemlevel analysis that focuses on complex interactions within biological systems ${ }^{[6]}$. Carcinogenesis is a complicated process of cell transformation by a progression of changes at the cellular, genetic and epigenetic levels ${ }^{[7,8]}$. Therefore, systems biology might shed light on the molecular mechanisms of the anticancer effects of TCMs, which may inspire the rethinking of the modern treatment of cancer.

Compound Kushen Injection (CKI) is a mixture of natural compounds extracted from two medical herbs: Kushen (Radix Sophorae Flavescentis) and Baituling (Rhizoma Smilacis Glabrae). Kushen has a long history of use for the treatment of solid tumors, inflammation and other diseases ${ }^{[1]}$. The primary compounds found in CKI are matrine, oxymatrine and sophoridine $^{[9,10]}$. Here we will introduce the anti-cancer effects of matrine and oxymatrine because there is little research about sophoridine. 


\section{Anti-cancer effects of matrine}

As one of the major alkaloids of CKI, matrine has been well documented to have anti-tumor effects in different cancer cells, including breast cancer cell lines (MCF-7), gastric cancer cells (SGC-7901 and MKN45), gallbladder carcinoma cells (GBC$\mathrm{SD})$, osteosarcoma tumor cells (UMR-108), and liver cancer cells (HepG2) ${ }^{[11]}$. All of these studies indicated that matrine could inhibit cancer cell proliferation and induce apoptosis via different molecular pathways.

Matrine was reported to effectively inhibit the proliferation of breast cancer cells, including ER-positive MCF cells, HER2positive BT-474 cells and highly metastatic MDA-MB-231 cells. The death of cancer cells was observed in the matrine treatment group. The expression of the inhibitor of $\kappa \mathrm{B}(\mathrm{I} \kappa \mathrm{B})$ kinase $\beta$ (IKK $\beta$ ) was downregulated by matrine in the NF-kB signaling pathway ${ }^{[12]}$. Another study indicated that matrine might suppress breast cancer cell (MCF-7) growth by inducing apoptosis and cell cycle arrest at the $G_{1} / S$ phase in a dose- and time-dependent manner via the miR-21/PTEN/Akt pathway. Matrine might repress the expression of miR-21, which can target and down-regulate the tumor suppressor PTEN. The upregulation of PTEN dephosphorylates Akt, resulting in an accumulation of Bad, p21/WAF1/CIP1 and p27/KIP1, which trigger intrinsic apoptotic cascades ${ }^{[13]}$.

Matrine can also inhibit cell proliferation and induce apoptosis in a dose- and time-dependent manner in gastric carcinoma cells (SGC-7901). The expression of Fas/Fasl and the activity of caspase- 3 enzyme increased after treatment with matrine, and these changes were highly correlated with the apoptosis rate ${ }^{[14]}$. Another study used matrine to treat human gastric cancer cells (MKN45). In that study, matrine activated the caspase- 3 and -7 pathways and increased the pro-apoptotic molecules of the Bcl-2 family (Bok, Bak, Bax, Puma, and Bim) to inhibit cell proliferation and induce apoptosis in a dosedependent manner ${ }^{[15]}$.

The treatment of human gallbladder carcinoma cells (GBCSD) with matrine was associated with an inhibition of cancer cell growth, induction of $\mathrm{G}_{1}$ cell cycle arrest and activation of apoptosis in a dose- and time-dependent manner. Apoptosis was activated by the down-regulation of the anti-apoptotic protein $\mathrm{Bcl}-2$ and the upregulation of the pro-apoptotic protein Bax after the cancer cells were treated with matrine ${ }^{[16]}$.

The dose- and time-dependent inhibition of cell growth and the induction of apoptosis were also observed in rat osteosarcoma UMR-108 cells (bone tumor) after treatment with matrine. It has been demonstrated that matrine can disrupt the mitochondrial transmembrane potential and upregulate reactive oxygen species. The downregulation of $\mathrm{Bcl}-2$ and the upregulation of Bax were observed in matrine-treated UMR108 cells, in which apoptosis was subsequently activated ${ }^{[17]}$.

In addition to the functional roles of inhibiting cell proliferation and inducing $\mathrm{G}_{1}$-phase cell cycle arrest and apoptosis that have been reported in other cancer cells, matrine can also activate autophagy in human HepG2 cells in a dose-dependent manner. After treatment with matrine, abundant cytoplasmic vacuoles of different sizes were observed and vacuolization progressively became larger and denser with the increase of concentration of matrine in HepG2 cells. The results from treatment with the specific autophagic inhibitor 3-MA by electron microscopy confirmed the effect of matrine in inducing autophagy ${ }^{[18]}$. The anti-tumor effect of matrine on murine hepatocellular carcinoma $\mathrm{H} 22$ cells was also demonstrated, and the inhibition of cell proliferation and the activation of apoptosis were proposed as the likely mechanisms ${ }^{[19]}$.

Matrine can also inhibit cell viability and induce cell apoptosis in human acute myeloid leukemia (AML) cells in a doseand time-dependent manner. After treatment with matrine, apoptosis in AML cells was induced by collapsing the mitochondrial membrane potential, inducing cytochrome $c$ release from mitochondria, reducing the ratio of $\mathrm{Bcl}-2 / \mathrm{Bax}$, increasing the activation of caspase-3, and decreasing the levels of p-Akt and $\mathrm{p}$-ERK $1 / 2^{[20]}$. In K562 cells, matrine was shown to inhibit cell proliferation and induce apoptosis via the mitochondrial pathway in a time- and dose-dependent manner. Analyses showed that E2F-1 and Apaf-1 were upregulated and that caspase- 9 and -3 were activated after the exposure to matrine ${ }^{[21]}$.

Li et al ${ }^{[22]}$ identified 128 miRNAs exhibiting >2-fold expression changes in matrine-treated cells relative to their expression levels in untreated cells. The data revealed that the majority of the 57 identified enrichment pathways were highly involved in tumorigenesis.

\section{Anti-cancer effects of oxymatrine}

Oxymatrine is another major alkaloid component extracted from the roots of Kushen. Studies have suggested that oxymatrine has anti-cancer effects on different cancer cells, including breast cancer cells (MCF-7), human pancreatic cancer cells (PANC-1), gastric cancer cells (SGC-7901, MKN-45, MKN-74), and human liver cancer cells (SMMC-7721).

The treatment of breast cancer cells (MCF-7) with oxymatrine was associated with an inhibition of cell proliferation and a decrease in the number of stem cell-like side population (SP) cells. The anti-cancer effect of oxymatrine on MCF-7 cells may be due to the inhibition of the $\mathrm{Wnt} / \beta$-catenin signaling pathway ${ }^{[23]}$. Oxymatrine can also induce apoptosis in human pancreatic cancer PANC-1 cells by a mechanism similar to matrine. After treatment with oxymatrine, livin and survivin genes were down-regulated and the $\mathrm{Bax} / \mathrm{Bcl}-2$ ratio was increased, accompanied by the release of mitochondrial cytochrome $c$ and the activation of caspase-3 protein in PANC-1 cells $^{[24]}$. It is also suggested that oxymatrine combined with angiogenesis inhibitor NM-3 has a synergistic inhibitory effect on the growth of human gastric cancer SGC-7901, MKN-45, and MKN-74 cells in a time-dependent manner.

Semi-quantitative PCR analyses showed that oxymatrine in combination with NM-3 can induce apoptosis by downregulating the expression of Survivin and Bcl-2 and upregulating the expression of p53 mRNA ${ }^{[25]}$. The downregulation of the bcl-2 gene and the upregulation of the p53 gene were also observed in human hepatoma cells after treatment with oxymatrine. A significant cell cycle blockage in the $G_{2} / M$ and $S$ phases was detected in oxymatrine-treated human hepatoma SMMC-7721 
cells, which might explain the inhibition of cell proliferation ${ }^{[26]}$.

\section{Studies on Compound Kushen Injection}

CKI has been widely used in clinical trials, and there are many clinical reports demonstrating its anti-cancer effect. These reports include using CKI to treat gastric cancer, liver cancer, lung cancer, breast cancer, ovarian cancer, colorectal cancer and other cancer types ${ }^{[1]}$.

Recently, the mechanisms of CKI used as anti-cancer therapy were investigated. The in vitro and in vivo analyses of CKI in human breast cancer MCF-7 cells showed that CKI could inhibit the growth of MCF-7 stem-like SP cells. The size of SP cells was suppressed, and the primary genes of the Wnt signaling pathway, such as $\beta$-catenin, cyclinD1, and c-Myc, were downregulated after treatment with CKI. This may also explain how CKI can attenuate the side effects of chemotherapy; chemotherapeutic drugs only inhibit non-SP cells (differentiated cells), not cancer stem-like cells (SP cells) ${ }^{[26]}$. In another study, CKI had an anti-inflammatory effect, protecting against carcinogen-induced oxidative damage, which inhibits rat gastric carcinogenesis ${ }^{[27]}$.

CKI has a significant anti-tumor effect on $\mathrm{H} 22$ and hepatic cancer, Lewis lung cancer and S180 tumors. In addition, it has synergistic and attenuation actions with anti-tumor agents in H22 and S180 cancer cells. The anti-tumor mechanisms of CKI may include: (1) increased protein expression of p16; (2) reduced unmethylated state of the p16 gene; (3) inhibited VEGF and MVD expression in neoplastic tissues; (4) decreased microvessel density ${ }^{[11,27,28]}$. Zhou et al ${ }^{[28]}$ investigated the effects of CKI on gastric immunity and oxidantantioxidant status during $N$-methyl- $N$ '-nitro- $N$-nitrosoguanidine (MNNG)-induced gastric carcinogenesis. They found that the administration of CKI significantly enhanced serum IgA, IgG, IgM, IL-2, IL-4, and IL-10 levels; decreased serum IL-6 and TNF-a levels; lowered the levels of lipid peroxides; and enhanced glutathione (GSH) levels and activities of GSHdependent enzymes. The authors suggested that CKI blocked experimental gastric carcinogenesis by protecting against carcinogen-induced oxidative damage and improving immunity activity.

Zhao et al state that CKI is a traditional Chinese medicine used alone or with chemotherapy to reduce cancer-associated pain. CKI treatment limited mouse sarcoma growth both in vivo and in vitro, in part by reducing the phosphorylation of ERK, AKT kinases and BAD. CKI inhibited TRPV1-mediated capsaicin-induced ERK phosphorylation and reduced tumorinduced pro-inflammatory cytokine production (Figure 1). Thus, CKI limited cancer pain both directly by blocking TRPV1 signaling and indirectly by reducing tumor growth ${ }^{[29]}$.

The efficacy of CKI has been evaluated in cancer patients either alone or in conjunction with chemotherapeutic or radiotherapeutic treatments. Shao et al reported that CKI combined with radiotherapy could significantly reduce adverse effects and improve the quality of life, the symptoms and the efficacy in elderly esophageal cancer patients ${ }^{[30]}$. Recently, Sun et al observed that CKI plus transcatheter arterial chemoemboliza-

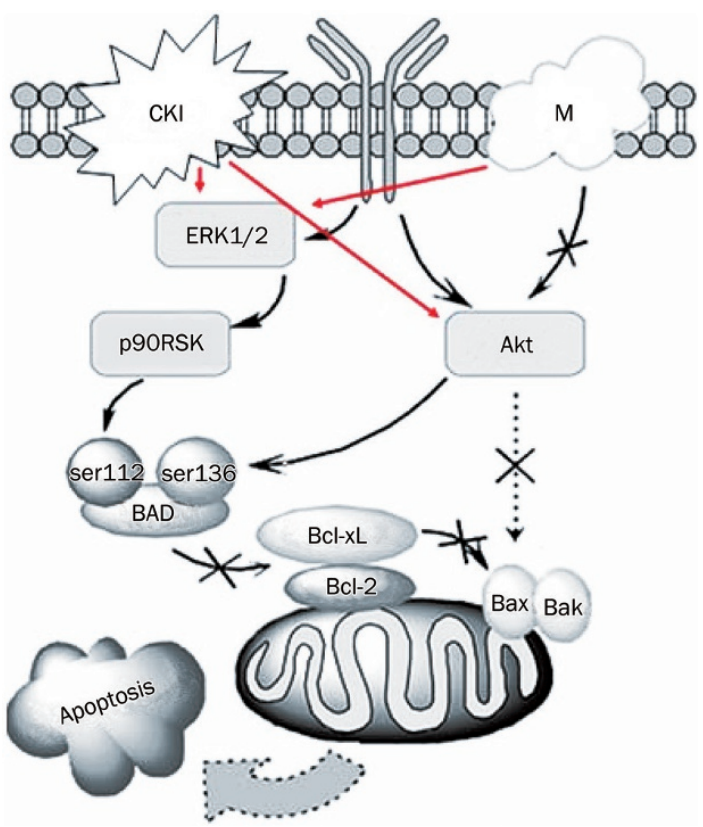

Figure 1. Compound Kushen injection (CKI) limited mouse sarcoma growth both in vivo and in vitro by reducing the phosphorylation of ERK, AKT kinases, and BAD.

tion (TACE) is superior to TACE alone. They suggested that CKI could be a complementary drug with TACE to inhibit the growth of hepatocellular carcinoma, suppress tumor metastasis, reduce the incidence of pain and improve the quality of life in patients with unresectable hepatocellular carcinoma ${ }^{[31]}$. Bao et al have conducted a systematic literature research, which revealed a positive effect of CKI on bone cancer pain: compared with radiotherapy or bisphosphonates, CKI showed significant effects on the improvement of pain relief in patients with bone cancer pain and the increase in Karnofsky scores (KPS). The patients treated with CKI achieved statistically significant reductions in the incidence of leukopenia and nausea. No severe adverse events were found and no treatment was stopped because of adverse events of CKI in the treatment groups $^{[32]}$.

Many TCMs are a mixture of different natural compounds extracted from multiple medical herbs. In the past, most studies only focused on one or several extracted compounds. We have not seen studies using system-wide approaches to understand the molecular mechanisms of TCMs such as CKI. We hypothesize that the molecular mechanism underling the anticancer effect of CKI might involve altering gene regulation in several pathways.

\section{Acknowledgements}

This work was supported by the Medical Scientific Research Foundation of Guangdong Province, China (2013B2013236). We thank Ms Zhi-zheng ZHAO in the Guang An Men Hospital of the China Academy of Chinese Medical Sciences for her kindly help in drawing the figure. 


\section{References}

1 Sun FG, Sun XY. "Sophora flavescens", in Shen Nong Ben Cao Jing. Scientific and Technical Documents Publishing House; 1999. p59.

2 Commission P. Pharmacopoeia of the People's Republic of China 2010 English Edition 2011: Chinese Pharmacopoeia Commission.

3 Bai MS, Wu ZP, Wang XC. Advances in research on adjuvant effects applying Traditional Chinese Medicine in cancer chemotherapy. J Modern Oncol 2010; 18: 597-601.

4 Li J, Lin HS, Hou W, Hua BJ. Idea and strategy of traditional Chinese medicine treatment for cancer. China Cancer 2010; 19: 735-8.

5 Yin SY, Wei WC, Jian FY, Yang NS. Therapeutic applications of herbal medicines for cancer patients. Evid Based Complement Alternat Med 2013; 2013: 302426.

6 Kitano H. Systems biology: a brief overview. Science 2002; 295: 1662-4.

7 Croce CM. Oncogenes and cancer. N Engl J Med 2008; 358: 50211.

8 Kanwal R, Gupta S. Epigenetic modifications in cancer. Clin Genet 2012; 81: 303-11.

9 Tian J, Wang WH, Gao HM, Wang ZM. Determination of matrine, sophoridine and oxymatrine in Compound Kushen Injection by HPLC. Zhongguo Zhong Yao Za Zhi 2007; 32: 222-4.

10 Qi L, Zhang J, Zhang Z. Determination of four alkaloids in Compound Kushen Injection by high performance liquid chromatography with ionic liquid as mobile phase additive. Se Pu 2013; 31: 249-53.

11 Sun M, Cao H, Sun L, Dong S, Bian Y, Han J, et al. Antitumor activities of kushen: literature review. Evid Based Complement Alternat Med 2012; 2012: 373219.

12 Shao H, Yang B, Hu R, Wang Y. Matrine effectively inhibits the proliferation of breast cancer cells through a mechanism related to the NF-KB signaling pathway. Oncol Lett 2013; 6: 517-20

13 Li LQ, Li XL, Wang L, Du WJ, Guo R, Liang HH, et al. Matrine inhibits breast cancer growth via miR-21/PTEN/Akt pathway in MCF-7 cells. Cell Physiol Biochem 2012; 30: 631-41.

14 Dai ZJ, Gao J, Ji ZZ, Wang XJ, Ren HT, Liu XX, et al. Matrine induces apoptosis in gastric carcinoma cells via alteration of Fas/FasL and activation of caspase-3. J Ethnopharmacol 2009; 123: 91-6.

15 Luo C, Zhu Y, Jiang T, Lu X, Zhang W, Jing Q, et al. Matrine induced gastric cancer MKN45 cells apoptosis via increasing pro-apoptotic molecules of Bcl-2 family. Toxicology 2007; 229: 245-52.

16 Zhang Z, Wang X, Wu W, Wang J, Wang Y, Xu X, et al. Effects of matrine on proliferation and apoptosis in gallbladder carcinoma cells (GBC-SD). Phytother Res 2012; 26: 932-7.

17 Yan F, Liu Y, Wang W. Matrine inhibited the growth of rat osteosarcoma UMR-108 cells by inducing apoptosis in a mitochondrialcaspase-dependent pathway. Tumor Biol 2013; 34: 2135-40.

18 Zhang JQ, Li YM, Liu T, He WT, Chen YT, Chen XH, et al. Antitumor effect of matrine in human hepatoma $G_{2}$ cells by inducing apoptosis and autophagy. World J Gastroenterol 2010; 16: 4281-90.
19 Ma L, Wen S, Zhan Y, He Y, Liu X, Jiang J. Anticancer effects of the Chinese medicine matrine on murine hepatocellular carcinoma cells. Planta Med 2008; 74: 245-51.

20 Zhang S, Zhang Y, Zhuang Y, Wang J, Ye J, Zhang S, et al. Matrine induces apoptosis in human acute myeloid leukemia cells via the mitochondrial pathway and akt inactivation. PLoS One 2012; 7 : e46853.

21 Jiang $\mathrm{H}$, Hou C, Zhang S, Xie H, Zhou W, Jin Q, et al. Matrine upregulates the cell cycle protein E2F-1 and triggers apoptosis via the mitochondrial pathway in K562 cells. Eur J Pharmacol 2007; 559: 98-108.

22 Li H, Xie S, Liu X, Wu H, Lin X, Gu J, et al. Matrine alters microRNA expression profiles in SGC-7901 human gastric cancer cells. Oncol Rep 2014; 32: 2118-26.

23 Zhang Y, Piao B, Zhang Y, Hua B, Hou W, Xu W, et al. Oxymatrine diminishes the side population and inhibits the expression of betacatenin in MCF-7 breast cancer cells. Med Oncol 2011; 28 Suppl 1: S99-107.

24 Ling Q, Xu X, Wei X, Wang W, Zhou B, Wang B, et al. Oxymatrine induces human pancreatic cancer PANC-1 cells apoptosis via regulating expression of $\mathrm{Bcl}-2$ and IAP families, and releasing of cytochrome $\mathrm{C}$. J Exp Clin Cancer Res 2011; 30: 66.

25 Song MQ, Zhu JS, Chen JL, Wang L, Da W, Zhu L, et al. Synergistic effect of oxymatrine and angiogenesis inhibitor NM-3 on modulating apoptosis in human gastric cancer cells. World J Gastroenterol 2007; 13: 1788-93.

26 Song G, Luo Q, Qin J, Wang L, Shi Y, Sun C. Effects of oxymatrine on proliferation and apoptosis in human hepatoma cells. Colloids Surf B Biointerfaces 2006; 48: 1-5.

27 Xu W, Lin H, Zhang Y, Chen X, Hua B, Hou W. Compound Kushen Injection suppresses human breast cancer stem-like cells by downregulating the canonical Wnt/beta-catenin pathway. J Exp Clin Cancer Res 2011; 30: 103.

28 Zhou SK, Zhang RL, Xu YF, Bi TN. Antioxidant and immunity activities of Fufang Kushen Injection Liquid. Molecules 2012; 17: 6481-90.

29 Zhao Z, Fan H, Higgins T, Qi J, Haines D, Trivett A, et al. Fufang Kushen injection inhibits sarcoma growth and tumor-induced hyperalgesia via TRPV1 signaling pathways. Cancer Lett 2014; 355: 232-41.

30 Shao Q. The recent effect of radiotherapy combined with compound kushen injection for esophageal cancer. Radiother Oncol 2011; 99 : S372-3.

31 Sun Q, Ma W, Gao Y, Zheng W, Zhang B, Peng Y. Meta-analysis: therapeutic effect of transcatheter arterial chemoembolization combined with compound kushen injection in hepatocellular carcinoma. Afr J Tradit Complement Altern Med 2012; 9: 178-88.

32 Bao Y, Yang L, Hua B, Hou W, Zhan S, Li W, et al. A systematic review and meta-analysis on the use of traditional Chinese medicine compound kushen injection for bone cancer pain. Support Care Cancer 2014; 22: 825-36. 